\title{
How can MINOCA be diagnosed? An anecdotal case reports
}

\author{
Massimo Bolognesi* \\ Internal General Medicine - GP's, AUSL della Romagna - District of Cesena, Via Ungaretti 49447521 Cesena, Italy
}

\begin{abstract}
Nowadays, normal or unobstructed coronaries in patients presenting at ED with chest pain and troponin positivity occurs more frequently in the emergency department, especially in women. A definite diagnosis may prove elusive and long-term management of such patients poses more difficult questions. Cardiac Magnetic Resonance imaging may prove to be useful in most of such situations by providing a certain or most likely diagnosis, but particularly by excluding noncardiac etiologies. MINOCA pathophysiology is still poorly understood which makes its diagnostics and treatment challenging in everyday clinical practice. The aim of this case report is to assess and discuss the clinical presentation and characteristics of a MINOCA patient in the setting of inconclusive cardiovascular imaging evidence.
\end{abstract}

\section{Introduction}

The ESC working group has well established the diagnostic criteria for MINOCA syndrome [1]. The diagnosis of MINOCA requires the following points.

1. Clinical and/or biochemical evidence of acute MI with increased biomarkers for myocardial injury and electrocardiographic changes,

2. The exclusion of obstructive coronary artery disease as defined by coronary stenosis $<50 \%$ (either invasively or with computed tomography angiography), and no other apparent cause for the acute presentation

Therefore, clinical history, electrocardiogram (ECG), specific cardiac enzymes, echocardiography, coronary and left ventricular angiography, are the first line of diagnostic investigation to identify the causes of MINOCA [2]. MINOCA might be just an initial and general diagnosis which does not describe underlying pathophysiology. The potential pathophysiological mechanisms of a clinical scenario where Myocardial Infarction (MI) may be diagnosed according to the definition, are much more complex considered that the main cause of MI is obstructive coronary disease. These include both coronary and non-coronary pathologies. The coronary causes comprise several different mechanisms. Thromboembolism itself may be an underlying pathological factor or may be caused by plaque rupture or coronary spasm. This includes also thrombotic disorders (hereditary or acquired) [3].

Plaque disruption may be caused by erosion, ulceration, plaque rupture, and intraplaque haemorrhage. Coronary artery spasm may be present not only due to endogenous causes but may be provoked by exogenous substances like cocaine [4,5]. Non-coronary aetiologies are also frequent in MINOCA patients. It is important to recognize welldefined diseases with described aetiologies like myocarditis, pulmonary embolism or Takotsubo cardiomyopathy in patients initially described as MINOCA.

MINOCA patients represent a conundrum given the many possible aetiologies, but the most likely underlying mechanism could be an epicardial coronary spasm or unstable coronary plaques not revealed or not understood by angiography. Coronary artery spasm may potentially contribute to the pathogenesis of AMI in patients without obstructive $\mathrm{CAD}$ and particularly warrants close consideration in those with MINOCA. This reflects a vascular smooth muscle hyperreactivity to endogenous vasospastic substances (as in vasospastic angina) but may also occur in the context of exogenous vasospastic agents. Microvascular spasm is also a potential cause of MINOCA since elevated troponins have been detected via ultrasensitive assays following provocative spasm testing, despite the absence of inducible large vessel spasm [6].

Its pathophysiology is poorly understood which makes diagnostics and treatment of MINOCA challenging in everyday clinical practice. The aim of this case report was to assess and discuss the clinical presentation and characteristics of MINOCA patient in a context of inconclusive cardiovascular imaging evidence.

\section{Case Report}

A 58-year-old female, with no prior medical history, presented to the ED for sudden nocturnal sharp chest pain radiated to the back. Her first ECG resulted quite normal (Figure 1), while blood analysis found out specific highly sensitive Troponin over normal limits ( $82 \mathrm{ng} / \mathrm{L}$ first check, then 400). Additionally, a fast echocardiogram showed marked hypokinesia at the mid-apical segment of left ventricular septal wall. She was immediately treated for Acute Coronary Syndrome (ACS) with beta-blocker, venitrin infusion, antiaggregant and anticoagulation therapy with pain relief and stable hemodynamics. Then, the patient

${ }^{\star}$ Correspondence to: Massimo Bolognesi, Internal General Medicine - GP's, AUSL della Romagna - District of Cesena, Via Ungaretti 49447521 Cesena, Italy, Tel: +390547645074; E-mail: massimo.bolognesi@medici.progetto-sole.it

Key words: MINOCA, cardiac resonance magnetic imaging, electrocardiogram, heart disease

Received: March 13, 2019; Accepted: March 24, 2019; Published: March 29, 2019 
was taken urgently to the catheterization lab where she was found to have to have a right hypoplastic coronary artery and a dominant left coronary artery free of atherosclerotic obstructive disease (Figure 2). The ECG of the day after (Figure 3), taken during the recovery in the cardiology department, showed significant changes in the ST-segment and T-wave in the precordial anteroseptal leads which suggested ischemia. Anyway, a few days later she was discharged in good health from the hospital with a diagnosis of suspected myocarditis. A follow-up ECG done at the GP's office (Figure 3) showed that the significant changes recorded in the cardiology department were more accentuated, confirming ST-segment elevation and Negative T-waves in the precordial anteroseptal leads. Also, a TTE confirmed a slight anteroseptal hypokinesis. Subsequently, 15 days later a CMR was performed to rule out myocarditis or other pathologies with myocardial damage, as both cardiologists and GP's have suspected. However, radiologists reported that no signs of myocarditis were shown by the CMR and there was no mention of myocardial damage like oedema or scar or signs of microvascular obstruction (MVO), even though some images (Figure 4) could suggest oedema on the Antero-mid-septal and apical wall suitable for microvascular obstruction compatible with the ECG findings and clinical presentation.

\section{Discussion}

The discussion aims to highlight the diagnostic difficulties that regard this novel syndrome despite the fact the clinical criteria are well defined and established. Vascular dysfunction without obstructive coronary disease is more prevalent in women and associated with increased use of healthcare resources, higher symptom burden, and importantly 2 -fold higher mortality, indicating that non-obstructive coronary disease is not always a benign condition [7,8]. Simply put, this case describes the presentation of acute myocardial infarction with ECG changes and troponin positivity without obstructive coronary artery disease and with CMR not diriment for MINOCA syndrome. In fact, as clinical history demonstrates, the patient was first admitted to the emergency department for signs of an acute coronary syndrome that was promptly suspected for the rise of specific troponin, and for the echocardiographic finding of hypokinesia of the mid and apical segment septal wall, even though the ECG resulted apparently normal. Consequently, the patient was taken to the hemodynamic laboratory to undergo her to coronary angiography, which however showed epicardial coronary arteries free from obstructive disease. The next inpatient cardiology period ran regularly and after a few days, the patient was discharged from the hospital with the diagnosis of suspected



Figure 1. Normal ECG recorded at the Emergency Department

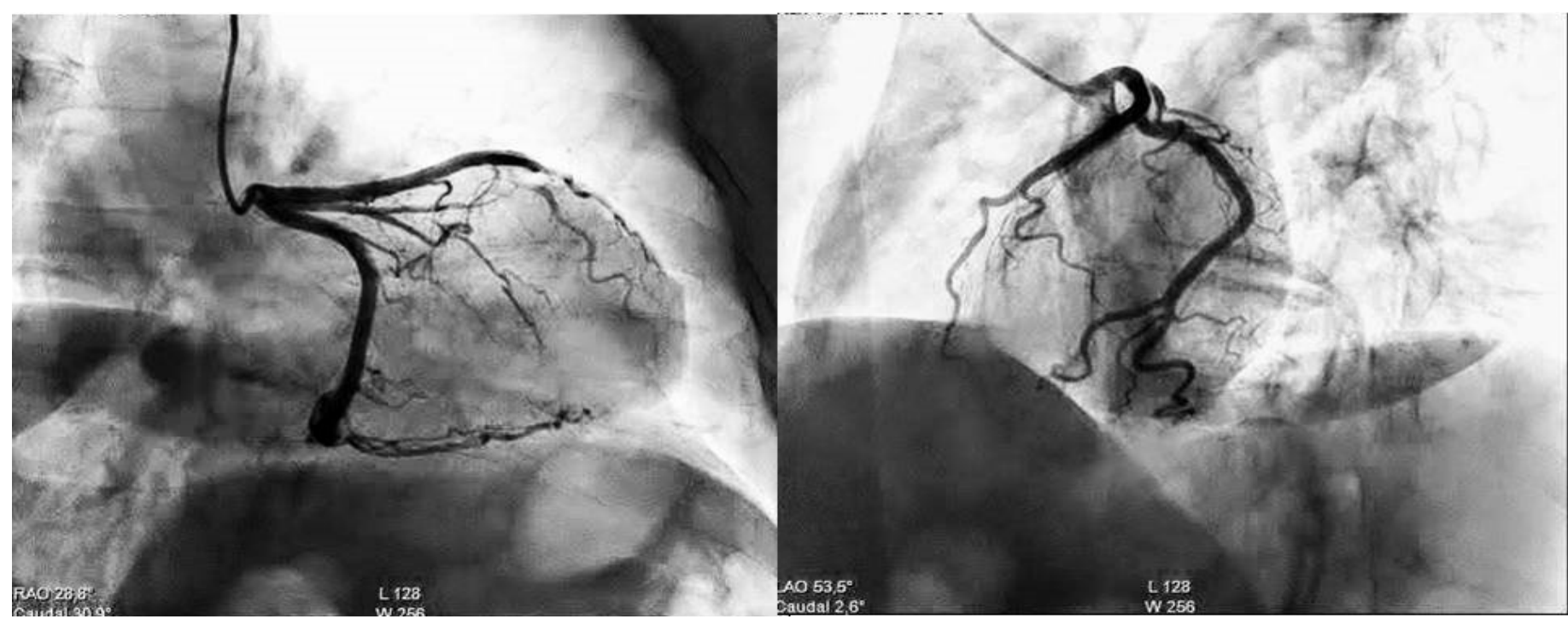

Figure 2. Coronary angiography with NON-obstructive CAD 

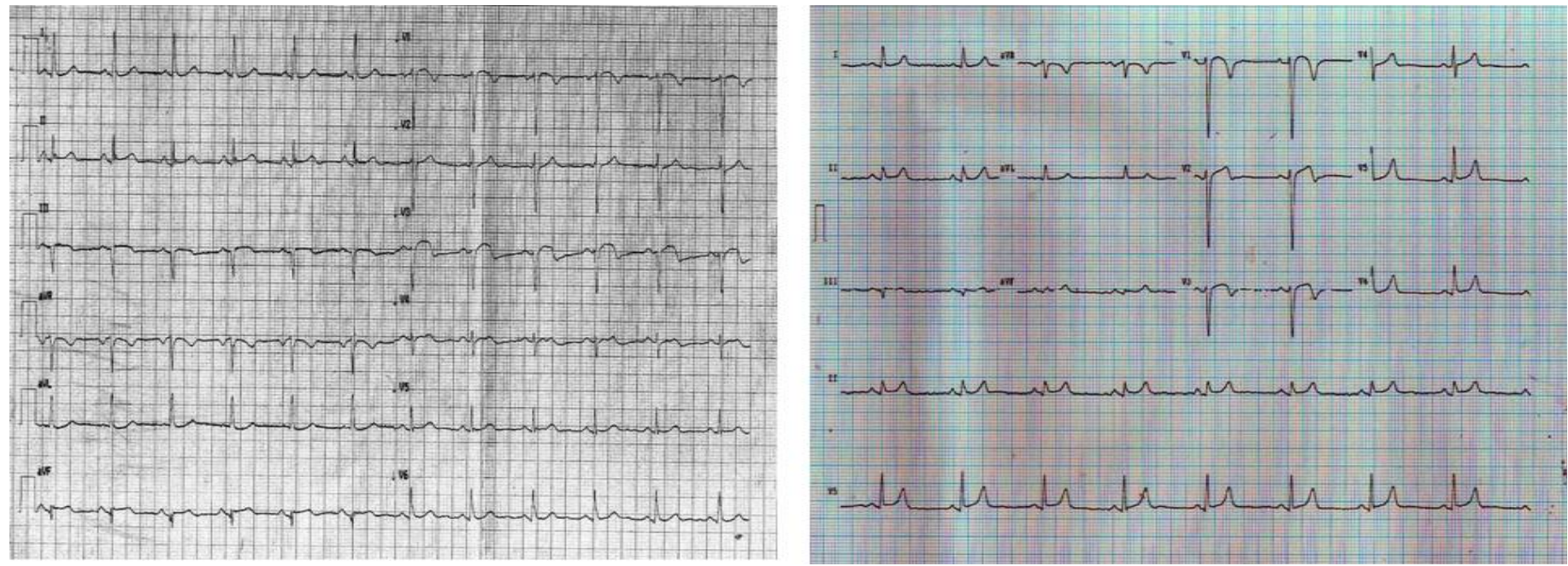

Figure 3. Follow up ECGs recorded at the Cardiology dept (on the left side) and at GP's office (on the right side) both showing ST-segment elevation and inverted T Waves in the anteroseptal leads


Figure 4. CMR findings that showed a spot of microvascular obstruction (MVO) in anteroseptal and apical wall

myocarditis. However, the follow-up ECG showed clear changes when compared to the ECGs performed in the ED and cardiology department, showing an ECG epicardial pattern of anteroseptal wall ischemia. A subsequent cardiac magnetic resonance imaging (CMR) performed 15 days after the onset of the symptoms appeared at first negative reading for myocarditis and indicated just a slight dilatation of the left cardiac chambers. Even so, an in-depth view of the CMR images showed some suggestive aspects on the apical septal wall compatible with minimal myocardial damage with isolated MVO spots. It is therefore plausible that the patient had MINOCA cardiac syndrome (i.e. coronary spasm, partial occlusion quickly revascularized, etc.) without serious consequence thanks to the prompt drug therapy performed. The diagnostic and prognostic impact of cardiovascular magnetic resonance imaging plays a key role in assessing patients with suspected myocardial infarction with unobstructed coronary arteries.
Therefore, CMR is strongly recommended by various experts and the ESC's task force when evaluating patients with the working diagnosis of MINOCA. In any case, CMR is recommended within 7 days of presentation because delayed imaging can sometimes result in some features no longer being evident [9].

Yet, as literature reported, there are cases of MINOCA with apparently negative CMR. At this regard, a recent study [10] has shown that in a large cohort of patients with MINOCA, CMR (median 37 days from presentation) identified a final diagnosis in $74 \%$ of patients. Thus, in more than $1 / 4$ of the patients, the CMR may show no specific or characteristic signs of myocardial infarction. In this case, the diagnosis of MINOCA was essentially clinical because of the clear evidence of a gradual change in ECG from normal to pathological with a specific and significant increase in the troponin in absence of obstructive coronary 
artery disease, even though the CMR had not been conclusive. The timing of CMR may be a factor and finding an alternate diagnosis for the rise in troponin becomes relevant. To date, no studies have addressed this population, and their management remains unclear. It follows that there is still much to do and study on these not straightforward and equivocal clinical situations, so quite difficult to diagnose even in the modern era of cardiovascular imaging.

\section{Disclosure}

No conflict of interest to declare.

\section{References}

1. Agewall S, Beltrame JF, Reynolds HR, Niessner A, Rosano G, et al. (2016) ESC working group position paper on myocardial infarction with non-obstructive coronary arteries. Eur Heart J 38: 143-53. [Crossref]

2. Niccoli G, Scalone G, Crea F (2015) Acute myocardial infarction with no obstructive coronary atherosclerosis: mechanisms and management. Eur Heart $J$ 36: 475-481. [Crossref]

3. Pasupathy S, Air T, Dreyer RP, Tavella R, Beltrame JF (2015) Systematic review of patients presenting with suspected myocardial infarction and nonobstructive coronary arteries. Circulation 131: 861-870. [Crossref]
4. Ramphul K, Mejias SG, Joynauth (2018) Cocaine, amphetamine, and cannabis use increases the risk of acute myocardial infarction in teenagers. Am J Cardiol 123: 354. [Crossref]

5. Kaski JC, Crea F, Meran D, Rodriguez L, Araujo L, et al. (1986) Local coronary supersensitivity to diverse vasoconstrictive stimuli in patients with variant angina. Circulation 74: 1255-1265. [Crossref]

6. Arrebola-Moreno AL, Arrebola JP, Moral-Ruiz A, Ramirez-Hernandez JA, MelgaresMoreno R, et al. (2014) Coronary microvascular spasm triggers transient ischemic left ventricular diastolic abnormalities in patients with chest pain and angiographically normal coronary arteries. Atherosclerosis 236: 207-214. [Crossref]

7. D'Onofrio G, Safdar B, Lichtman JH, Strait KM, Dreyer RP, et al. (2015) Women are less likely than men to be diagnosed with cardiovascular disease early and to receive an appropriate and timely intervention, including pharmacotherapy. Circulation 131: 1324-1332. [Crossref]

8. Madonis SM, Skelding KA, Roberts M (2017) Management of acute coronary syndromes: special considerations in women. Heart 103: 1638-46. [Crossref]

9. Pasupathy S, Tavella R, McRae S, Beltrame JF (2015) Myocardial Infarction with NonObstructive Coronary Arteries - Diagnosis and Management. Eur Cardiol 10: 79-82. [Crossref]

10. Dastidar AG, Baritussio A, De Garate E, Drobni Z, Biglino G, et al. (2019) Prognostic Role of Cardiac MRI and Conventional Risk Factors in Myocardial Infarction with Nonobstructed Coronary Arteries. JACC: Cardiovascular Imaging S1936-878X: 30065-30068. [Crossref]

Copyright: ( 02019 Bolognesi M. This is an open-access article distributed under the terms of the Creative Commons Attribution License, which permits unrestricted use, distribution, and reproduction in any medium, provided the original author and source are credited. 